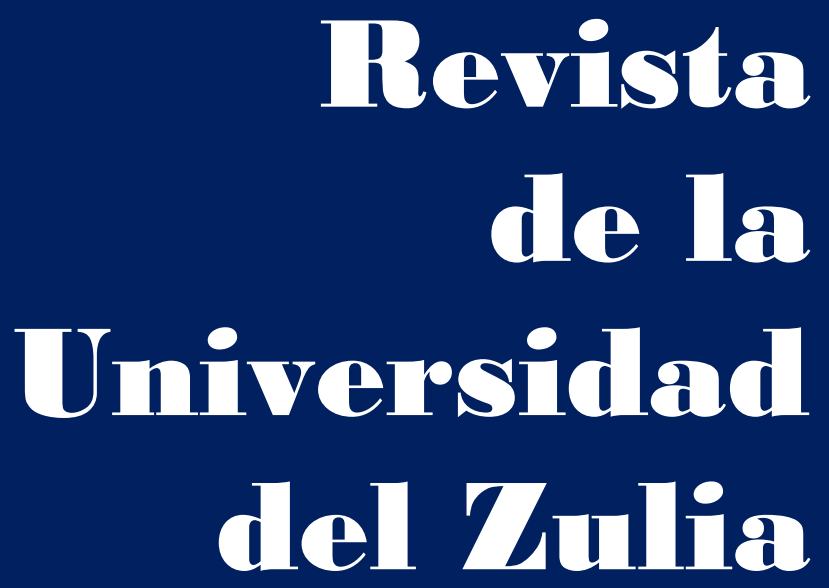

Fundada en 1947

por el Dr. Jesús Enrique Lossada

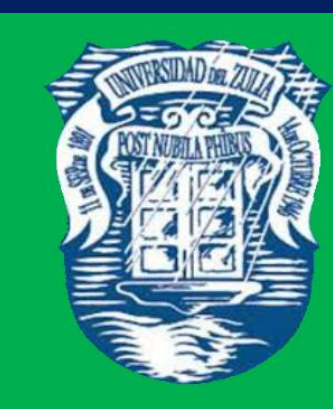

Ciencias del

Agrad,

Ingemiería

y Tecinología

\section{Aกัต 13 No $\mathbf{3 6}$} Enero - Abril 2022

Tercera Épaca

Maracailbo-Venezuela 


\title{
La certificación Rainforest Alliance como estrategia competitiva para el sector agrícola exportador ecuatoriano
}

\author{
Henry Junior Moncada Roblez * \\ Jonella Abigail Guzmán Armijos ** \\ Mayiya Lisbeth González Illescas *** \\ Sandra Sayonara Solorzano Solórzano ****
}

\section{RESUMEN}

Las transformaciones que experimentan los mercados internacionales son una constante que deben enfrentar las empresas para su expansión comercial, poniendo a prueba su capacidad de respuesta y adaptación a los nuevos escenarios. Para cumplir con las estrictas regulaciones y ganar la confianza de los consumidores, los exportadores están adoptando diferentes tipos de certificación como estrategia para gestionar la sostenibilidad en mercados exigentes. Este trabajo se plantea como objetivo identificar la influencia de la certificación Rainforest Alliance en la competitividad de las empresas exportadoras ecuatorianas en el mercado internacional. El análisis se enfoca en varios productos del sector agrícola. Se ha empleado el método teórico analítico-sintético para revisar artículos en revistas científicas y documentos bibliográficos de instituciones oficiales. Entre los principales resultados se destaca que esta certificación contribuye de forma significativa a la protección del medio ambiente, la equidad y la viabilidad económica de la empresa. Se concluye que al aplicar este sello ambiental se obtienen beneficios en la fincas o empresas aumentando la producción permitiendo el acceso a nuevos mercados.

PALABRAS CLAVE: Comercio Internacional; desarrollo sostenible; estrategia de diferenciación; Rainforest Alliance.

*Docente. Universidad Técnica de Machala, Ecuador. ORCID: https://orcid.org/0000-00018803-5248. E-mail: hmoncadal@utmachala.edu.ec

**Docente. Universidad Técnica de Machala, Ecuador. ORCID: https://orcid.org/0000-00015994-8822. E-mail: jguzman4@utmachala.edu.ec

***Docente. Universidad Técnica de Machala, Ecuador. ORCID: https://orcid.org/0000-00025219-3807. E-mail: mlgonzalez@utmachala.edu.ec

****Docente. Universidad Técnica de Machala, Ecuador. ORCID: https://orcid.org/0000-00016294-7396. E-mail: ssolorzano@utmachala.edu.ec

Recibido: 01/11/2021

Aceptado: 20/12/2021 


\section{Rainforest Alliance certification as a competitive strategy for the Ecuadorian agricultural export sector}

ABSTRACT

The transformations that international markets are undergoing are a constant that companies must face for their commercial expansion, testing their ability to respond and adapt to new scenarios. To comply with strict regulations and win the trust of consumers, exporters are adopting different types of certification as a strategy to manage sustainability in demanding markets. The objective of this work is to identify the influence of the Rainforest Alliance certification on the competitiveness of Ecuadorian exporting companies in the international market. The analysis focuses on various products in the agricultural sector. The analyticalsynthetic theoretical method has been used to review articles in scientific journals and bibliographic documents of official institutions. Among the main results, it stands out that this certification contributes significantly to the protection of the environment, equity and the economic viability of the company. It is concluded that by applying this environmental seal, benefits are obtained in the farms or companies by increasing production, allowing access to new markets.

KEYWORDS: International Trade; sustainable development; differentiation strategy; Rainforest Alliance.

Introducción

El dinamismo que se observa en los mercados internacionales se asocia a los cambios de comportamiento que ha tenido el consumidor. Se destaca la preocupación por los efectos de actividades productivas en el medio ambiente y el desarrollo sostenible. Así, un creciente número de empresas reconocen mediante sus prácticas, la necesidad de adaptarse a las transformaciones derivadas del medio ambiente para mantenerse en la competencia.

Dentro de este marco se puede recalcar que las organizaciones han empezado a tomar acciones basadas en la responsabilidad social, puesto que la misma es un factor importante dentro de la competitividad en las empresas, de modo que esta comprende diversos campos que van desde los precios hasta la calidad del producto. En este sentido, las empresas han recurrido 
a diversas estrategias que van direccionadas al desarrollo sostenible, para poder incrementar su aceptación en el mercado.

Existe evidencia de empresas que se destacan por aplicar como estrategia de diferenciación la certificación Rainforest Alliance, que fomenta la acción colectiva entre el hombre y la naturaleza. Dicha certificación respalda empresas que emplean mecanismos efectivos para vincular a los pequeños agricultores de los países en desarrollo con mercados de alto valor (Sellare et al., 2020), generando patrones de producción y comercio respetuosos con el medio ambiente y socialmente aceptables, que permiten destacarse de forma significativa en el mercado competitivo al que se está expuesto, estableciendo estrategias basadas en el conocimiento de lo que desea el consumidor (Carro et al., 2019). La certificación Rainforest Alliance se enfoca en la protección del medio ambiente desde la producción hasta las cadenas de supermercados asegurando a los consumidores productos que han sido labrados y cosechados, cumpliendo con cada uno de sus estándares (Vera y Cañón, 2018) aportando valor a millones de agricultores y cientos de empresas a nivel mundial.

En Ecuador, existen empresas agrícolas exportadoras que han podido mantenerse a través del tiempo defendiendo su competitividad mediante la inclusión de la certificación Rainforest Alliance en las plantaciones de banano, café, bosques, aceite de palma, cacao, té, helechos y flores. Se destaca el sector florícola que, en el año 2018, registraba 45 empresas con la implementación de esta autenticación (Mena et al., 2018).

En la línea de lo expuesto, esta investigación se desarrolla enmarcada en el objetivo de identificar la influencia de la certificación Rainforest Alliance en la competitividad de las empresas exportadoras ecuatorianas en el mercado internacional. Para alcanzar el propósito, esta investigación se realizó bajo un enfoque descriptivo, a través del análisis de artículos de revistas científicas y escritos de instituciones oficiales asociados a varios sectores que integran al sector agrícola. Se emplearon los métodos teóricos analítico-sintético. La investigación se direcciona a dar respuesta a la interrogante ¿Cuáles son las oportunidades que brinda la certificación Rainforest Alliance a las empresas agrícolas exportadoras?

El presente trabajo se estructura a partir de la introducción, luego se expone en la revisión de literatura la relación entre la competitividad y las certificaciones tomando en cuenta 
fundamentos teóricos. Se indaga la situación de la certificación Rainforest Alliance en el sector agrícola exportador ecuatoriano. Después de hacer la descripción de la metodología, se presentan los resultados enfocados a la eficacia de las certificaciones en los mercados internacionales y su impacto en las empresas agrícolas ecuatorianas. Se presentan las conclusiones enmarcadas en los impactos de los sellos de desarrollo sostenible como parte competitiva de las empresas agrícolas. Finalmente, se exponen los obstáculos y las propuestas de nuevas líneas de investigación a futuro.

\section{Revisión de Literatura}

\subsection{Estrategias de diferenciación para la competitividad del sector agrícola}

El término estrategia se asocia a la organización de las actividades y recursos de la empresa, empleando aquellas ventajas de las cuales el adversario carece (Weihrich y Koontz, 1993). A partir de los años 60 la aplicación de la estrategia en el ámbito empresarial conlleva el interés de varios autores considerados clásicos. Chandler (1962) y Andrews (1971) precisan que tanto la estrategia como la estructura empresarial deben estar acordes entre sí para asegurar una ejecución airosa en un tiempo prolongado. A su vez Ansoff (1965) asocia la estrategia al vínculo común que existe entre las actividades organizativas y las relaciones producto-mercado. Es así que, en el progreso de las perspectivas, Sallenave (1994) describe a la estrategia como el arte de vincular todos los aspectos de la gestión empresarial para buscar una mayor competitividad de modo que nos permita saber hacia dónde vamos y cómo obtenerlo.

Por esta razón, la definición de la estrategia en una organización puede ser la mejor apuesta para establecer un orden y asignación efectiva de recursos para lograr una situación viable (Mintzberg et a., 1997). La estrategia es una base fundamental en la proyección de la internacionalización (Heiss, 2017), considerando que procura una adaptación favorable ante los cambios que surgen en el mercado. Así, una forma de aprovechar esta oportunidad es utilizar factores diferenciadores en los productos o servicios para proporcionar un valor superior. En este contexto, en la tabla l que reúne propuestas manifestadas por diversos autores sobre la estrategia de diferenciación. 
REVISTA DE LA UNIVERSIDAD DEL ZULIA. 3르 época. Año 13 N$^{\circ}$ 36, 2022 Henry Junior Moncada Roblez et al. /// La certificación Rainforest Alliance como estrategia competitiva ... 7-27 DOI: http://dx.doi.org/10.46925//rdluz.36.02

Tabla l. Conceptualización de estrategia de diferenciación

\begin{tabular}{cl}
\hline \multicolumn{1}{c}{ Autor } & \multicolumn{1}{c}{ Descripción } \\
\hline (Job y Nyongesa, 2016) & $\begin{array}{l}\text { Las corporaciones requieren desarrollar estrategias que las } \\
\text { diferencien de sus competidores por ello necesitan de } \\
\text { capacidades superiores de ingeniería y diseño. Los diversos } \\
\text { grados de diferenciación comprenden cambiar los atributos } \\
\text { del producto, la marca y el empaque, etiquetado y ofrece } \\
\text { servicios distinguidos de soporte al producto. }\end{array}$ \\
\hline Las empresas que utilizan una estrategia de diferenciación \\
consideran la primera entrada al mercado como una prioridad \\
absoluta. Al ser la primera en el mercado, la empresa tiene la \\
discreción de establecer precios y explotar el amplio \\
segmento del mercado en la búsqueda de lograr altas \\
ganancias y crecimiento
\end{tabular}

Fuente: Elaboración propia a partir de los autores citados

La ejecución de la estrategia de diferenciación puede contribuir a mejorar la competitividad, ya que en el entorno globalizado la contienda entre las empresas se agudiza, de tal manera que el desarrollar características únicas en un producto o servicio se convierte en una capacidad indispensable. Porter (1980) destaca que en el ámbito de consecución se crea una posición provechosa a través de la dimensión básica de diferenciación que busca instaurar un conjunto de capacidades distintas, con atributos exclusivos e incomparables a los de su oposición. En este sentido el interés por analizar la estrategia de diferenciación se revela en los trabajos de los autores como Chirinos y Rosado (2016) quienes manifiestan que deben ser única y valiosa, llevando iniciativas como la planificadas a largo plazo (Mora et al., 2020) para lograr 
superioridad ante la competencia (Onufrey y Bergek, 2021; Pavlović y Čelić, 2020) con el fin de que la empresa se pueda distinguir dentro de su industria (Gallegos et al., 2020).

Al hilo de lo expuesto, la relación que existe entre la estrategia de diferenciación y la competitividad puede manifestarse a través de la innovación en productos y procesos, a la cual el público consumidor se muestra muy receptivo. Así, Bernal y Rodriguez (2019) precisan que al consolidarse la competitividad esta genera un crecimiento a nivel global como resultado de la satisfacción fundada por los usuarios.

En síntesis, a lo expuesto se asevera que las empresas pueden generar mayor competitividad adoptando estrategias que les permitan hacer evidente los factores diferenciadores entre sus contendientes. Por lo tanto, de acuerdo con los estándares internacionales, la diferenciación basada en modelos de gestión es una alternativa que están empleando las compañías exitosas en el mercado.

\subsection{La Certificación Rainforest Alliance como estrategia de diferenciación}

La certificación Rainforest Alliance es una organización creada en 1987, dirigida al sector agrícola sin fines de lucro. El sello está representado por una rana verde que permite al demandante reconocer la sostenibilidad económica, social y ambiental (Fantin y De Lara, 2020). Se encuentra asociado al compromiso con los tres pilares del desarrollo sostenible: mejora continua, sostenibilidad y rentabilidad para los agricultores (Rainforest Alliance, 2018), el cual busca conservar un equilibrio entre la existencia humana y los recursos naturales a través de prácticas de plantaciones amigables (Martinez García et al., 2017).

Siendo la protección de los recursos naturales un aspecto que recibe la atención de un creciente número de consumidores, la certificación Rainforest Alliance aporta de forma significativa en la credibilidad de la institución otorgándole un reconocimiento a nivel internacional, salvaguardando la confiabilidad del etiquetado frente a la competencia del mercado.

Rainforest Alliance afirma garantizar la salud a largo plazo de la comunidad al proteger el ecosistema, mantener el bienestar de la comunidad local y aumentar la productividad (Vanderhaegen et al., 2018). Asegurando medios de vida sostenibles a través de cambios en el uso 
de la tierra (Brenton, 2018) e instaurando un futuro mejor para las personas al hacer que los negocios responsables sean el nuevo modelo a seguir.

La cobertura que tiene esta certificación a nivel mundial se ve reflejada en todos los países, de tal manera, que en la actualidad se ve inmersa en 70 naciones. "Este sello se aplica principalmente en los cultivos de árboles (como el cacao, café), té, fruta (como los cocos, piñas y bananos), nueces (como las avellanas) y flores cortadas, verduras y palma, especialmente en tipo de organizaciones pequeñas y grandes" (Rainforest Alliance, 2020). Por consiguiente, al aplicar esta certificación su valor de referencia varía de acuerdo a la ubicación y tamaño de la finca, así como el producto que se vaya a certificar. En Ecuador la entidad que se encarga auditar, certificar y formar auditores en temas de sostenibilidad en los campos de agricultura bajo la certificación RA es Conservación y Desarrollo (C y D Certified S.A).

Varios autores entre ellos González (2018) y Caviedes et al. (2020) concuerdan en señalar que las empresas que adoptan de forma voluntaria la certificación Rainforest Alliance, están definiendo su estrategia para mejorar su desempeño ambiental y consolidar su prestigio en el acatamiento de rigurosos protocolos sobre el proceso de producción y la cadena de suministro. En este sentido, están empleando la certificación como una herramienta para entrar a nuevos mercados ya su vez mantener una posición competitiva frente a la demanda sustancial de productos sostenibles.

\section{Metodología}

La investigación es desarrollada con un enfoque cualitativo y de alcance descriptivo fundamentado en revisión de literatura, de acuerdo al objetivo planteado de identificar la influencia de la certificación Rainforest Alliance en la competitividad de las empresas exportadoras ecuatorianas en el mercado internacional.

Las bases de datos examinadas fueron Ebsco, Redalyc, Scopus, Scielo, Taylor \& Francis ScienceDirect y además del motor de exploración de Google Académico. De manera que, las indagaciones de información se ejecutaron por título, resumen y palabras claves, utilizando los siguientes términos de búsqueda en español, inglés y portugués, las cuales se enmarcaron en palabras como "evolución conceptual de la certificación RA", "aplicación y evolución de la 
certificación RA", "enfoque estratégico de RA para el logro de ventajas competitivas", "la certificación RA como estrategia de diferenciación", "estrategia de diferenciación como fuente de ventaja competitiva", "La certificación RA como estrategia para medirse en negocios internacionales" y "la certificación RA en empresas exportadoras".

En esta fase fue elemental la lectura rápida de la introducción de los artículos conseguidos, y los predefinidos se ordenan en la matriz de contenido bibliográfico. Se obtuvieron 40 artículos aceptados. La segunda estrategia de búsqueda comprendió la revisión y evaluación de los artículos previamente definidos entre los cuales constan 15 aceptaciones. Posteriormente, los métodos de lectura y análisis ayudaron a afinar las referencias bibliográficas obtenidas, es decir aquellos que cumplían con los siguientes criterios de inserción:

- Temática principal Rainforest Alliance

- El origen de la información debe estar comprendida por artículos de revisión, artículos científicos y libros

- Las publicaciones deben ser entre los años de 2016 y 2021, excepto para autores clásicos.

- En el apartado de resultados, las publicaciones deben presentar un diseño de investigación cuantitativo que aborde casos de empresas que mantengan vigente la aplicación de la certificación Rainforest Alliance para la obtención de ventajas competitivas.

En las evaluaciones de resultados literarios y las discusiones, se ha implementado el método teórico analítico-sintético e inductivo-deductivo. El análisis se logra extrayendo información relevante y asociando u ordenando la información obtenida por medio de la escritura se alcanza la síntesis. El método deductivo-inductivo se considera en la identificación de las variables que muestra la gestión estratégica de las organizaciones para fortalecer la ventaja competitiva del sector agrícola, en el ámbito de casos de empresas que han adquirido la certificación Rainforest Alliance.

\section{Resultados}

En base al objetivo planteado, se exponen los resultados de la investigación realizada, de acuerdo a la recopilación de trabajos que hacen mención a la certificación Rainforest Alliance, 
REVISTA DE LA UNIVERSIDAD DEL ZULIA. 3épeca. Año 13 Nº 36, 2022

Henry Junior Moncada Roblez et al. /// La certificación Rainforest Alliance como estrategia competitiva ... 7-27

DOI: http://dx.doi.org/10.46925//rdluz.36.02

bajo un enfoque descriptivo se expone en la tabla 2 la sistematización de 15 artículos científicos en la cual se obtiene información relevante como la muestra, el país de estudio y los principales resultados.

Tabla 2. Certificación Rainforest Alliance aplicada en las fincas o empresas exportadoras.

\begin{tabular}{|c|c|c|}
\hline Autor/Año & Muestra/País & Resultados \\
\hline (Munasinghe et al., 2021) & $\begin{array}{l}74 \text { productores de té. } \\
\text { (Sri Lankan) }\end{array}$ & $\begin{array}{l}\text { El sello Rainforest Alliance en los } \\
\text { catálogos de los corredores es } \\
\text { sumamente importante debido a que } \\
\text { permite crear un intercambio } \\
\text { económico desde los suministros de } \\
\text { las plantaciones de té con los } \\
\text { consumidores de mentalidad ética. } \\
\text { También permite que los } \\
\text { intermediarios observen la diferencia } \\
\text { de precios que genera la demanda de } \\
\text { consumidores, promoviendo a } \\
\text { implementar la certificación. }\end{array}$ \\
\hline (Dietz y Grabs, 2021) & $\begin{array}{l}659 \text { productores de café } \\
\text { hondureños. } \\
\text { (Honduras) }\end{array}$ & $\begin{array}{l}\text { Los agricultores certificados por } \\
\text { Rainforest Alliance tuvieron los } \\
\text { rendimientos más altos en cuanto a } \\
\text { precios, logrando así ingresos } \\
\text { significativos por hectárea de café. }\end{array}$ \\
\hline (Mora et al., 2020) & $\begin{array}{l}\text { Empresas exportadoras } \\
\text { y cinco productos del } \\
\text { sector agropecuario. } \\
\text { (Ecuador) }\end{array}$ & $\begin{array}{l}\text { Las certificaciones como RA cumplen } \\
\text { un gran papel debido a que } \\
\text { contribuyen a generar beneficios a las } \\
\text { empresas exportadoras ecuatorianas, } \\
\text { permitiendo incrementar sus ventas, } \\
\text { fidelizar clientes y lograr ingresar a } \\
\text { nuevos mercados. }\end{array}$ \\
\hline (Teopista et al., 2020) & $\begin{array}{l}454 \text { hogares agrícolas } \\
\text { productores de café, de } \\
\text { los cuales } 126 \text { cuentan } \\
\text { con certificación } \\
\text { Fairtrade, } 81 \text { con } \\
\text { certificación Rainforest } \\
\text { Alliance y } 247 \text { sin } \\
\text { certificación. } \\
\text { Etiopía y Uganda }\end{array}$ & $\begin{array}{l}\text { Los ingresos totales cafeteros y el ingreso } \\
\text { familiar per cápita son mayor para los } \\
\text { productores con certificación RA, en } \\
\text { comparación con los no certificados, esto } \\
\text { se da debido a los altos rendimientos } \\
\text { especialmente por la combinación de la } \\
\text { certificación RA con la certificación } 4 \mathrm{C} \text { y } \\
\text { Utz que se enfocan directamente en las } \\
\text { buenas prácticas agrícolas. } \\
\text { Los ingresos de los productores con } \\
\text { certificación FT son más altos (pero } \\
\text { menos que los de hogares con } \\
\text { certificación RA) en comparación con los } \\
\text { de productores no certificados en Etiopía. }\end{array}$ \\
\hline
\end{tabular}




\begin{tabular}{|c|c|c|}
\hline (Donovan et al., 2019) & $\begin{array}{l}3 \text { tipos de empresas } \\
\text { cafeteras en cada país } \\
\text { en total } 9 \text { empresas } \\
\text { cafeteras } \\
\text { (Guatemala, Honduras } \\
\text { y Nicaragua) }\end{array}$ & $\begin{array}{l}\text { Esta certificación ha ayudado a que los } \\
\text { productores vendan su café a } \\
\text { compradores exigentes debido a la } \\
\text { competencia que existe en el mercado } \\
\text { internacional, también permitió } \\
\text { demostrar la calidad del producto, } \\
\text { negociar precios, mejorar las opciones } \\
\text { de comercialización, reducir riesgos } \\
\text { en el mercado y aumentar el acceso a } \\
\text { servicios e insumos. }\end{array}$ \\
\hline (Annunziata et al., 2019) & $\begin{array}{l}\text { Sur de Italia en una } \\
\text { muestra de } 305 \\
\text { personas de entre } 18 \text { y } \\
26 \text { años. } \\
\text { (Italia) }\end{array}$ & $\begin{array}{l}\text { Existe un bajo consumo de alimentos } \\
\text { con marca sustentable especialmente } \\
\text { con la de RA debido a la etiqueta poco } \\
\text { visible mencionando los encuestados } \\
\text { que no han consumido tales productos } \\
\text { con estos sellos. En términos de } \\
\text { facilidad de interpretación, RA se } \\
\text { considera el más difícil de interpretar. }\end{array}$ \\
\hline (Martinez et al., 2017) & $\begin{array}{l}58 \text { encuestas } \\
\text { personalizadas a } \\
\text { caficultores certificados } \\
\text { con el RA de las } \\
\text { provincias comuneras } \\
\text { (Colombia) }\end{array}$ & $\begin{array}{l}\text { Los caficultores mencionaron en las } \\
\text { encuestas en un } 100 \% \text { que la } \\
\text { certificación ha permitido el aumento } \\
\text { de la rentabilidad y competitividad, } \\
\text { mejorando el precio por el producto, } \\
\text { mientras que el } 95 \% \text { mencionaron que } \\
\text { se ha creado una conciencia amigable } \\
\text { con el medio ambiente reflejándose en } \\
\text { la calidad de vida, producción y } \\
\text { calidad del producto. }\end{array}$ \\
\hline (Takahashi y Todo, 2017) & $\begin{array}{l}\text { Área de Prioridad } \\
\text { Forestal Regional de } \\
\text { Belete-Gera. Café } \\
\text { forestal } \\
\text { (Etiopía) }\end{array}$ & $\begin{array}{l}\text { En esta investigación se identificó que } \\
\text { los productores certificados con RA } \\
\text { vendieron el café a un precio más alto } \\
\text { entre un } 15 \% \text { y un } 20 \% \text { que el café } \\
\text { convencional. Las evaluaciones o } \\
\text { auditorías continuas podría ser un } \\
\text { factor que motivaría a los productores } \\
\text { certificados a conservar los bosques } \\
\text { debido al incentivo económico que } \\
\text { existe. }\end{array}$ \\
\hline (De Andrade et al., 2017) & $\begin{array}{l}126 \text { consumidores de } \\
\text { chocolate amargo } \\
\text { (Brasil) }\end{array}$ & $\begin{array}{l}\text { El estudio expresa que un alto } \\
\text { porcentaje de los encuestados considera } \\
\text { importante saber el origen del producto } \\
\text { ya que nunca han consumido chocolate } \\
\text { con sellos de calidad. De tal forma que el } \\
79 \% \text { está dispuesto a pagar un precio } \\
\text { mayor por el chocolate amargo } \\
\text { certificado. }\end{array}$ \\
\hline
\end{tabular}




\begin{tabular}{|c|c|c|}
\hline $\begin{array}{l}\text { (Teopista y Maertens, } \\
\text { 2017) }\end{array}$ & $\begin{array}{l}\text { Sector cafetero } \\
\text { (Uganda) }\end{array}$ & $\begin{array}{l}\text { Productores certificados por } \\
\text { Utz_RA_4C poseen una mayor } \\
\text { producción de café, rendimiento y } \\
\text { productividad laboral, el } 57 \% \text { del café } \\
\text { certificado tiene producción superior } \\
\text { frente a la no certificada. Este esquema } \\
\text { con las } 3 \text { certificaciones reduce la } \\
\text { probabilidad de ser pobres en } 16 \\
\text { puntos porcentuales, aumentando } \\
\text { tanto el ingreso familiar y el per cápita } \\
\text { que son efectos importantes en la } \\
\text { incidencia de la pobreza. }\end{array}$ \\
\hline (Mitiku et al., 2017) & $\begin{array}{l}\text { El sector cafetero del } \\
\text { suroeste de Etiopía } \\
\text { (Etiopía) }\end{array}$ & $\begin{array}{l}\text { Los agricultores con certificación RA } \\
\text { reciben un precio entre un } 40 \% \text { y un } \\
60 \% \text { más alto, teniendo ganancias } \\
\text { elevadas permitiendo mejorar el } \\
\text { bienestar de cada uno de ellos. En } \\
\text { comparación con la certificación FT y } \\
\text { Org tiene un mejor funcionamiento en } \\
\text { el impacto de los ingresos familiares y } \\
\text { reduciendo la pobreza. }\end{array}$ \\
\hline (Becerril, 2016) & $\begin{array}{l}\text { Sat Camposeven } \\
\text { (España) }\end{array}$ & $\begin{array}{l}\text { La certificación ecológica permite a } \\
\text { los productores ofrecer alimentos con } \\
\text { calidad reforzados por las } \\
\text { certificaciones internacionales dando } \\
\text { a conocer al consumidor el origen del } \\
\text { mismo, ayudando a que los productos } \\
\text { con sellos ecológicos se diferencian de } \\
\text { los demás influyendo en los clientes } \\
\text { que desean consumir alimentos sanos } \\
\text { potenciando la imagen empresarial en } \\
\text { los mercados extranjeros. }\end{array}$ \\
\hline (Calderón et al.,2014) & $\begin{array}{l}\text { Se seleccionaron } 36 \\
\text { fincas certificadas con } \\
\text { el sello Rainforest } \\
\text { Alliance por un período } \\
\text { mínimo de } 3 \text { años. } \\
\text { (Colombia) }\end{array}$ & $\begin{array}{l}\text { Las condiciones de vida en las fincas } \\
\text { de Cundinamarca y Santander han } \\
\text { mejorado gracias a la certificación RA, } \\
\text { aumentando la calidad del producto, } \\
\text { reduciendo las quejas de trabajadores } \\
\text { e incrementando la productividad y } \\
\text { mejorando la parte financiera. La } \\
\text { certificación ayudó a los agricultores a } \\
\text { una mejor exposición en el momento } \\
\text { de la venta, diferenciación del } \\
\text { producto, precios Premium y acceso } \\
\text { mejorado al crédito. }\end{array}$ \\
\hline (Guedes et al., 2014) & $\begin{array}{l}55 \text { fincas individuales y } \\
11 \text { grupos }\end{array}$ & $\begin{array}{l}\text { Tanto las fincas individuales y } \\
\text { grupales de café certificados por RA } \\
\text { tienen niveles comparables de }\end{array}$ \\
\hline
\end{tabular}


DOI: http://dx.doi.org/10.46925//rdluz.36.02

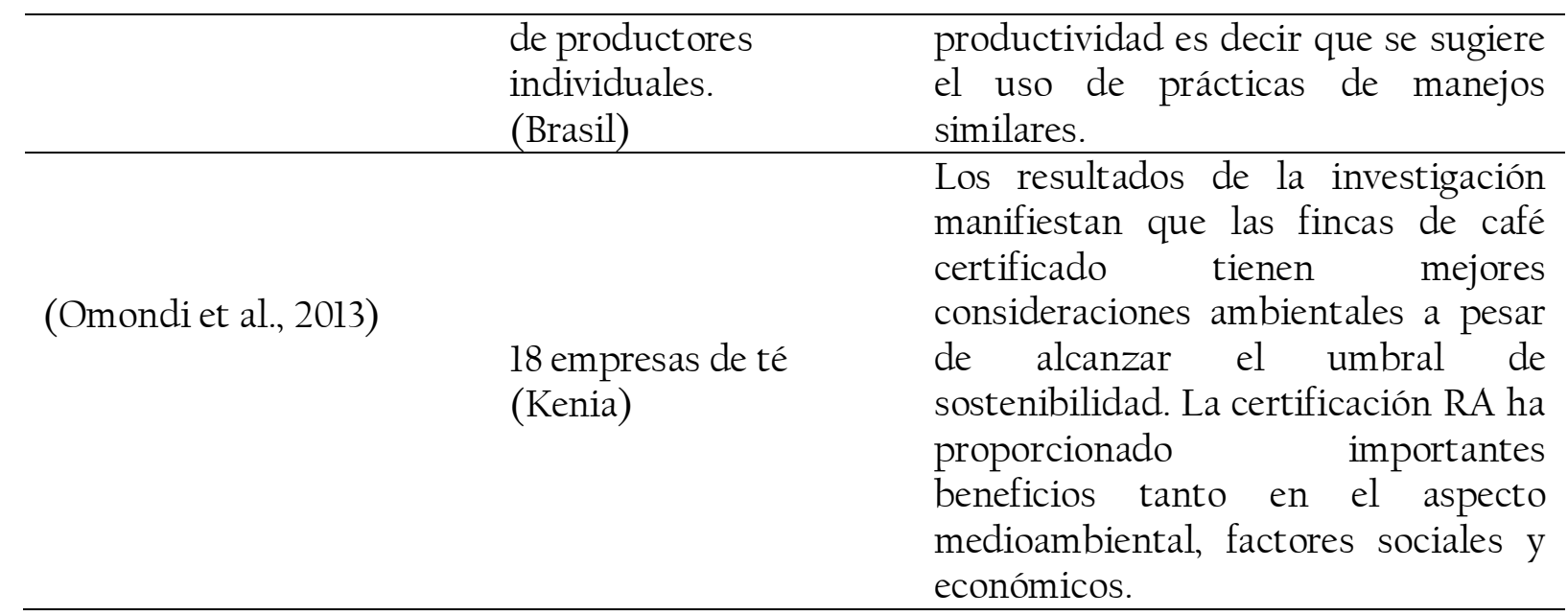

Fuente: Elaboración propia a partir de los autores citados

De la recopilación extraída se puede observar que los principales resultados manifiestan que al aplicar la certificación RA han obtenido muchos beneficios tanto para productores, fincas y trabajadores. Desde el enfoque de Calderón et al. ( 2014) se puede apreciar que al certificarse con este sello les ha permitido tener exposición en el mercado, precios más elevados, mejor ambiente laboral tanto productor como trabajador, aumento en la producción y acceso a créditos.

Según Mora et al. (2020) manifiestan que las certificaciones incluida la RA contribuyen en el acceso a nuevos mercados, fidelizando clientes y aumentando las ventas, también la certificación RA ha permitido vender a compradores muy exigentes en la parte de la calidad del producto, negociar precios y tener acceso a servicios e insumos permitiendo ser más competitivos en el mercado exterior (Donovan et al., 2019).

En la tabla 3 se presenta el alcance que ha tenido la certificación y el crecimiento relativo del periodo 2019-2020, en base a los productos como el café cacao, té y banano a nivel internacional.

Como lo menciona en sus informes Rainforest Alliance y UTZ (202la) el mayor crecimiento relativo lo ha tenido el café en el número de productores con un 18\% seguido por un $15 \%$ en el aumento de la producción estimada, también se puede notar un decrecimiento en las hectáreas de producción del cacao con un 33\% por ende el número de productores y la producción estimada bajó en un 25\%, sin embargo el número de trabajadores se elevó a un 8\% 
mientras que en el té hubo un crecimiento en casi todos los ítems del alcance del programa, a excepción del número de trabajadores manteniéndose en un 0\%.

Tabla 3. Alcance del programa Rainforest Alliance y el crecimiento relativo

\begin{tabular}{ccccccccc}
\hline $\begin{array}{c}\text { Alcance del } \\
\text { programa }\end{array}$ & Café & Cacao & Té & Banano & \multicolumn{3}{c}{ Crecimiento Relativo 2019-2020 } \\
\cline { 6 - 9 } & & & & Café & Cacao & Té & Banano \\
\hline $\begin{array}{c}\text { Área } \\
\text { (hectáreas) }\end{array}$ & 519,828 & 544,207 & 643,184 & 189,227 & $+10 \%$ & $-33 \%$ & $+5 \%$ & $+2 \%$ \\
\hline $\begin{array}{c}\text { Número de } \\
\text { productores }\end{array}$ & 228,430 & 156,391 & 958,528 & 2,123 & $+18 \%$ & $-25 \%$ & $+2 \%$ & $-3 \%$ \\
\hline $\begin{array}{c}\text { Producción } \\
\text { estimada } \\
\text { (TM) }\end{array}$ & 770,295 & 327,915 & $1,367,875$ & $9,462,755$ & $+15 \%$ & $-25 \%$ & $+12 \%$ & $+2 \%$ \\
\hline $\begin{array}{c}\text { Número de } \\
\text { trabajadores } \\
\text { (Temporales }\end{array}$ & 334,449 & 8,880 & 734,120 & 184,954 & $+14 \%$ & $+8 \%$ & $0 \%$ & $+2 \%$ \\
$\begin{array}{c}\text { y } \\
\text { permanentes) }\end{array}$ & & & & & & & \\
\hline $\begin{array}{c}\text { Ventas } \\
\text { mundiales }\end{array}$ & 403,241 & 186,005 & 375,283 & $9,462,755$ & $+2 \%$ & $-8 \%$ & $+2 \%$ & $+2 \%$ \\
\hline
\end{tabular}

Fuente: Adaptado a partir de Rainforest Alliance y UTZ (202lc)

Elaborado: por los autores

Las ventas mundiales se puede notar un crecimiento del 2\% en el café, té y banano más sin embargo el cacao tuvo un decrecimiento del $8 \%$ debido a los cambios de demanda y al COVID-19 (Rainforest Alliance y UTZ, 202lb).

A continuación, en la tabla 4 y 5 se presentan la participación de Ecuador con la certificación Rainforest Alliance en el mercado mundial.

En base a lo expuesto en la tabla 4 se puede observar que el mayor crecimiento relativo 2019-2020 ha sido el cacao teniendo un 53\% en ventas mundiales sin embargo existe un decrecimiento en la producción estimada en un $28 \%$ al igual que el té mientras que el banano presenta un incremento del $11 \%$.

En la tabla 5 se presentan las fincas certificadas con el programa de Rainforest Alliance en Ecuador en productos como banano, cacao, flores, rosas, aceite de palma, maracuyá, mango, piña, plátano, pitahaya y té. 
REVISTA DE LA UNIVERSIDAD DEL ZULIA. 3르 época. Año 13 N 36, 2022 Henry Junior Moncada Roblez et al. /// La certificación Rainforest Alliance como estrategia competitiva ... 7-27 DOI: http://dx.doi.org/10.46925//rdluz.36.02

Tabla 4. Participación de Ecuador con la certificación Rainforest Alliance

\begin{tabular}{lccccccc}
\hline \multirow{1}{*}{ Alcance de Ecuador } & Cacao & Té & Banano & \multicolumn{3}{c}{ Crecimiento Relativo 2019- } \\
\cline { 5 - 7 } & & & & Cacao & Té & Banano \\
\hline $\begin{array}{l}\text { Ventas mundiales según su } \\
\text { origen (TM) }\end{array}$ & 12,688 & 3,408 & 338,281 & $+53 \%$ & & - \\
\hline $\begin{array}{l}\text { Producción estimada según } \\
\text { su origen }\end{array}$ & 21,260 & 3,233 & $1,275,686$ & $-28 \%$ & $-13 \%$ & $+11 \%$ \\
\hline
\end{tabular}

Nota. Ecuador está considerado con otros países en la venta de té, pese a ello no refleja información del crecimiento relativo al igual que el banano.

Fuente: Adaptado a partir de Rainforest Alliance y UTZ (202lc)

Elaborado: por los autores

En la tabla 5 se presenta las fincas certificadas con Rainforest Alliance en Ecuador, de las cuales la mayoría se encuentran en las provincias de Guayas (68), El Oro (60), Los Ríos (55) y Pichincha (44), las mismas que en su mayoría son banano y cacao y flores mientras que los productos como piña, mango, maracuyá, pitahaya, té, rosas y aceite de palma son las que tienen un menor número de fincas certificadas. Cabe recalcar que la única provincia certificada por parte de la región Oriental es Morona Santiago, con dos productos diferentes como la pitahaya y el té. Las provincias del Chimborazo y Santa Elena son las únicas que tienen una finca certificada. Es decir, en Ecuador se cuenta con un total de 270 fincas certificadas con RA.

Tabla 5. Fincas certificadas con el programa Rainforest Alliance en Ecuador.

\begin{tabular}{lccc}
\hline \multicolumn{1}{c}{ Provincia } & Tipo de producto & Número de fincas & Total, de fincas certificadas \\
\hline \multirow{2}{*}{ Cañar } & Banano & 3 & 4 \\
\hline Chimborazo & Cacao & 1 & 1 \\
\hline \multirow{5}{*}{ Cotopaxi } & Banano & 1 & 10 \\
& Blores & 7 & \\
& Banana & 1 & \\
\hline
\end{tabular}


REVISTA DE LA UNIVERSIDAD DEL ZULIA. 3ㄹépoca. Año 13 N$^{\circ}$ 36, 2022 Henry Junior Moncada Roblez et al. /// La certificación Rainforest Alliance como estrategia competitiva ... 7-27

DOI: http://dx.doi.org/10.46925//rdluz.36.02

\begin{tabular}{|c|c|c|c|}
\hline \multirow{2}{*}{ ElOro } & Banano & 57 & \multirow{2}{*}{60} \\
\hline & Cacao & 3 & \\
\hline \multirow{2}{*}{ Esmeraldas } & Aceite de Palma & 1 & \multirow{2}{*}{5} \\
\hline & Cacao & 4 & \\
\hline \multirow{5}{*}{ Guayas } & Banano & 60 & \multirow{5}{*}{68} \\
\hline & Maracuyá & 1 & \\
\hline & Cacao & 5 & \\
\hline & Flores & 1 & \\
\hline & Mango & 1 & \\
\hline Imbabura & Flores & 3 & 3 \\
\hline \multirow{4}{*}{ Los Ríos } & Banano & 44 & \multirow{4}{*}{55} \\
\hline & Cacao & 9 & \\
\hline & Mango & 1 & \\
\hline & Piña & 1 & \\
\hline \multirow{3}{*}{ Manabí } & Plátano & 2 & \multirow{3}{*}{8} \\
\hline & Cacao & 4 & \\
\hline & Banano & 2 & \\
\hline \multirow{2}{*}{$\begin{array}{l}\text { Morona } \\
\text { Santiago }\end{array}$} & Pitahaya & 1 & \multirow{2}{*}{2} \\
\hline & Té & 1 & \\
\hline \multirow{2}{*}{ Pichincha } & Flores & 38 & \multirow{2}{*}{44} \\
\hline & Rosas & 6 & \\
\hline Santa Elena & Banano & 1 & 1 \\
\hline \multirow{3}{*}{$\begin{array}{l}\text { Santo } \\
\text { Domingo }\end{array}$} & Piña & 4 & \multirow{3}{*}{9} \\
\hline & Banano & 3 & \\
\hline & Cacao & 2 & \\
\hline Total & & & 270 \\
\hline
\end{tabular}

Fuente: Adaptado a partir de (CyD, 2021)

Elaborado: por los autores 
Conclusiones

El presente trabajo se desarrolló bajo la perspectiva de abordar la certificación Rainforest Alliance como una estrategia de diferenciación que posibilita a las empresas exportadoras ecuatorianas generar una ventaja competitiva en el mercado internacional a través de las buenas prácticas ambientales aportando al desarrollo sostenible y a la concientización del consumidor. El certificado de calidad en la empresa es una buena medida para aprovechar su alto volumen de producción e ingresar al comercio exterior (Botello, 2016) adquiriendo un plus diferenciador a través de RAC, lo que permite ser competitivos donde existen estándares meticulosos para validar el producto (Morán et al., 2019).

Mediante el análisis de la certificación RA en la participación de los productos ecuatorianos se ha podido identificar que el café es uno de los productos agrícolas que no se encuentra certificado con este sello, sin embargo, podemos encontrar un alto número de fincas certificadas siendo el té, la pitahaya, maracuyá, mango, piña, aceite de palma y plátano uno de los productos no tradicionales que implementan dicha certificación para garantizar sus procesos, logrando distinguir de forma única en el mercado competitivo. También se puede observar que varias fincas alrededor del mundo han logrado mejorar su capacidad de producción, disminuir riesgos, perfeccionar el ambiente laboral, acceder a créditos financieros basados en tres principios esenciales como son la mejora continua, desarrollo sostenible y una buena utilidad para los campesinos.

En base al objetivo planteado en la investigación, se concluye que las empresas exportadoras implementan la estrategia de diferenciación mediante estándares de sostenibilidad que representa el sello, dado que el mercado es muy exigente, por ende, las organizaciones se han ido adaptando a estos cambios, ofertando productos de calidad desde su producción hasta su consumo otorgándole satisfacción al demandante.

El estudio y evaluación de las estadísticas de los diversos productos dan a conocer el crecimiento de la certificación en Ecuador de manera que aporta un incentivo para las pequeñas y grandes empresas a implementar el sello en su mercancía como elemento diferenciador que lo hace único de la competencia. 
La principal limitación de esta investigación se asocia a su enfoque cualitativo de carácter descriptivo, en donde se utilizaron las publicaciones de otros autores que examinaron el contexto de las empresas de diferentes sectores productivos, y países, de modo que las deducciones no podrían ser generalizadas a la realidad de otras empresas. Para las investigaciones futuras se deberían considerar estudios estadísticos correlacionales que permitan determinar el tipo de relación entre la variable de la estrategia de diferenciación (fundamentada en la RA) y la variable de la ventaja competitiva.

\section{Referencias}

Andrews, K. R. (1971). The concept of corporate strategy. Homewood, Ill., Dow Jones-Irwin.

Annunziata, A., Mariani, A., \& Vecchio, R. (2019). Effectiveness of sustainability labels in guiding food choices: Analysis of visibility and understanding among young adults. Sustainable Production and Consumption, 17, 108-115. https://doi.org/10.1016/j.spc.2018.09.005

Ansoff, I. (1965). The Corporate Strategy. New York, USA: MCGraw Hill.

Becerril Hernández, H. (2016). La certificación ecológica; una dualidad agrícola: Mejorar la prosperidad del agricultor y medio ambiente. Agroproductividad, 9(12), 45-50. https://revistaagroproductividad.org/index.php/agroproductividad/article/view/860

Bernal Jiménez, M. C., \& Rodriguez Ibarra, D. L. (2019). Las tecnologías de la información y comunicación como factor de innovación y competitividad empresarial. Scientia et Technica, 24(1), 85-95. https://doi.org/10.22517/23447214.20401

Botello Peñaloza, H. A. (2016). Las certificaciones de calidad y la internacionalización de las firmas industriales colombianas. Suma de Negocios, 7(16), 73-81. https://doi.org/10.1016/j.sumneg.2016.02.009

Brenton, S. (2018). Consumers and certification schemes: The ethics of global production and trade. Journal of Agricultural and EnvironmentalEthics, 31(6), 755-784. https://doi.org/10.1007/s10806018-9754-3

Brüning Larsen, S., Masi, D., Jacobsen, P., \& Godsell, J. (2018). How the reverse supply chain contributes to a firm's competitive strategy: a strategic alignment perspective. Production Planning \&Control, 29(6), 452-463. https://doi.org/10.1080/09537287.2017.1390178 
Calderón Cuartas, P. A., Trejos Pinzón, J. F., Serna Giraldo, C. A., \& Cruz Cerón, G. (2014). Certificación Rainforest Alliance, una mirada desde la percepción de los caficultores de Cundinamarca y Santander. Cenicafé, 7-22. https://biblioteca.cenicafe.org/handle/10778/482

Carro Suárez, J., Sarmiento Paredes, S., \& Rosano Ortega, G. (2019). Modelo de certificación para la dimensión institucional de desarrollo sustentable. Acta universitaria, 29. https://doi.org/10.15174/au.2019.2030

Caviedes Rubio, D. I., \& Olaya Amaya, A. (2020). Impacto ecológico, social y económico de fincas certificadas en buenas prácticas agrícolas y comercio justo. Cuadernos de Desarrollo Rural 17. https://doi.org/10.11144/Javeriana.cdr17.iese

Chandler, A. D. (1962). Strategy and Structure: Chapters in the history of American Enterprise. Boston: MIT Press.

Chirinos Cuadros, C. R., \& Rosado Samaniego, J. F. (2016). Estrategia de diferenciación: el caso de las empresas industriales. Ingeniería Industrial, 034, 165-174. https://doi.org/10.26439/ing.ind2016.n034.1342

CyD. (2021, Mayo 07). CYD Certified S.A. Retrieved from CYD Certified S.A.: https://cydcertified.com/documentos/index.php/documentos-publicos/clientes-certificados

De Andrade Silva, A. R., Sodré Bioto, A., Efraim, P., \& de Castilho Queiroz, G. (2017). Impact of sustainability labeling in the perception of sensory quality and purchase intention of chocolate consumers. Journal of Cleaner Production, 141, 11-21. https://doi.org/10.1016/j.jclepro.2016.09.024

Dietz, T., \& Grabs, J. (2021). Additionality and Implementation Gaps in Voluntary Sustainability Standards. New Political Economy, 1-22. https://doi.org/10.1080/13563467.2021.1881473

Donovan, J., Blare, T., \& Peña, M. (2019). Multiple certification uptake by coffee businesses: Evidence of functions and benefits from Central America. Business Strategy \& Development, 1-13. https://doi.org/10.1002/bsd2.93

Fantin Soares, F., \& De Lara Hungaro, O. A. (2020). A certificação Rainforest Alliance em uma propriedade rural no município de Tangará da Serra-MT. Revista de Empreendedorismo e Inovação Sustentáveis, 6(l), 5l-68. $\quad 6$ Retrieved from http://revista.isaebrasil.com.br/index.php/EGS/article/view/92/71

Gallegos, M. C., Beltrán, L. I., Calderón, L. C., \& Guerra, V. R. (2020). La diferenciación como estrategia de competitividad en el sector florícola del Cantón Cayambe (Ecuador). Revista Espacios, 41(10). Retrieved from https://www.revistaespacios.com/a20v4lnl0/a20v4lnl0p02.pdf 
REVISTA DE LA UNIVERSIDAD DEL ZULIA. 3르 época. Año 13 N 36, 2022

Henry Junior Moncada Roblez et al. /// La certificación Rainforest Alliance como estrategia competitiva ... 7-27 DOI: http://dx.doi.org/10.46925//rdluz.36.02

González Ordóñez, A. I. (2018). Las certificaciones ambientales ecuatorianas en la competitividad de las empresas. INNOVA Research Journal, 3(10.1), 55-67. https://doi.org/10.33890/innova.v3.n10.1.2018.785

Guedes Pinto , L. F., Gardner , T., McDermott , C. L., \& Lara Ayub , K. O. (2014). Group certification supports an increase in the diversity of sustainable agriculture network-rainforest alliance certified coffee producers in Brazil. Ecological Economics, 107, 59-64. https://doi.org/10.1016/j.ecolecon.2014.08.006

Heiss, G. (2017). Influencing Factors and the effect of organizational capabilities on internationalization strategies for German SMEs in the MedTech Industry. Management Studies, 5(4), 263-277. https://doi.org/ 10.17265/2328-2185/2017.04.001

Job, M., \& Nyongesa, M. B. (2016). Product differentiation strategy for competitive advantage in kenya cooperative creameries. Africa International Journal of Management Education, 1(3), 1-9. http://www.oircjournals.org/images/journals/product_differentiation_strategy_for_competitive _advantage.pdf

López Zapata, E., López Moros, G. P., \& Agudelo Muñoz, S. (2019). Relación entre Estrategias Competitivas y Tipos de Aprendizaje Organizativo en Empresas Colombianas. Información tecnológica, 30(5), 191-202. https://doi.org/10.4067/S0718-07642019000500191

Martinez García, J. D., Rios Vargas, C. F., \& Sanmiguel Jaimes, E. M. (2017). Evaluacion de la percepcion que tienen los caficultores frente la certificacion rainforest alliance de las provincias comuneras y guanenta, departamento de santader. Innovando En La U(9), 53-72. https://doi.org/10.18041/2216-1236/innovando.9.2017.3896

Mena Vásconez, P., Vos, J., Van Ommen, P., \& Boelens, R. (2018). Flores, acaparamiento del agua y responsabilidad empresarial social: certificación de la producción de rosas y reclamos por la justicia ambiental en el Ecuador. Cuadernos de geografía(101), 189-214. https://doi.org/10.7203/CGUV.101.13727

Méndez Naya, J. (2017). Fusiones horizontales de empresas y estrategias de diferenciación de producto. Estudios de Economía, 44(2), 45-58. https://doi.org/10.4067/S0718-52862017000200173

Mintzberg, H., Brian Quinn, J., \& Voyer, J. (1997). El proceso Estrategico: Conceptos, contextos y casos. México: Pearson Educación.

Mitiku, F., Maertens, M., De Mey, Y., \& Nyssen, J. (2017). Do Private Sustainability Standards Contribute to Income Growth and Poverty Alleviation? A Comparison of Different Coffee Certification Schemes in Ethiopia. Sustainability, 1-21. https://doi.org/10.3390/su9020246 
Mora Córdova, D. E., Lituma Loja, A. A., \& González Illescas, M. L. (2020). Las certificaciones como estrategia para la competitividad de las empresas exportadoras. INNOVA Research Journal, 5(2), 113-132. https://doi.org/10.33890/innova.v5.n2.2020.1274

Morán Montalvo, C. O., Cárdenas Zambrano, C. S., \& Elena, C. S. (2019). Característiccas de la responsabilidad social empresarial de las pymes en Ecuador. Caso de estudio: hacienda nueva colonia. Revista ECA Sinergia, 10(3), 131-144. https://doi.org/10.33936/eca_sinergia.vl0i3.1558

Munasinghe, A., Cuckston, T., \& Rowbottom, N. (2021). Sustainability certification as marketisation: Rainforest Alliance in the Sri Lankan tea production industry. Accounting Forum, 45, 247-272. https://doi.org/10.1080/01559982.2021.1893053

Omondi Ochieng, B., Hughey , K. F., \& Bigsby, H. (2013). Rainforest Alliance Certification of Kenyan tea farms: a contribution to sustainability or tokenism? Journal of Cleaner Production, 39, 285-293. https://doi.org/10.1016/j.jclepro.2012.07.048

Onufrey, K., \& Bergek, A. (2021). Transformation in a mature industry: The role of business and innovation strategies. Tecnovación, 105. https://doi.org/10.1016/j.technovation.2020.102190

Pavlović, N., \& Čelić, I. (2020). The analysis of competitive strategies from the perspective of small and medium enterprises. Hotel and Tourism Management, 8(1), 101-110. https:/doi.org/10.5937 / menhottur2001101P

Porter, M. E. (1980). Competitive strategy: techniques for analyzing industries and competitors. Free Press.

Rainforest Alliance. (2018, octubre 28). Retrieved from https://www.rainforestalliance.org/es/perspectivas/que-significa-rainforest-alliance-certified/

Rainforest Alliance. (2020, junio). Valores de Referencia de Salarios Dignos por país. Retrieved from https://www.rainforest-alliance.org/wp-content/uploads/2020/06/Anexo-10-Valores-deReferencia-de-Salarios-Dignos-por-pa\%C3\%ADs.pdf

Rainforest Alliance y UTZ. (2021a). Informe de Datos de Certificación del Café 2020. Programas de Rainforest Alliance y UTZ. Retrieved from https:/www.rainforest-alliance.org/es/resourceitem/informe-de-datos-de-certificacion-del-cafe-2020/

Rainforest Alliance y UTZ. (2021b). Informe de Datos de Certificación del Cacao 2020. Programas de Rainforest Alliance y UTZ.

Rainforest Alliance y UTZ. (202lc). Informe de datos de Certificación Rainforest Alliance 2020. Rainforest Alliance y UTZ. Programas de Rainforest Alliance y UTZ. Retrieved from https://www.rainforest-alliance.org/es/tag/certificacion-de-fincas/?fwp_by_resource=informes

Sallenave, J. P. (1994). La gerencia integral: no le tema a la competencia, témale a la incompetencia. Bogotá: Editorial Norma. 
Sellare, J., Meemken, E. M., Kouamé, C., \& Qaim, M. (2020). Do Sustainability Standards Benefit Smallholder Farmers Also When Accounting For Cooperative Effects? Evidence from Côte d'Ivoire. American Journal of Agricultural Economics, 102(2), 681-695. https://doi:10.1111/ajae.12015

Semuel, H., Siagian, H., \& Octavia, S. (2017). The Effect of Leadership and Innovation on Differentiation Strategy and Company Performance. Procedia - Social and Behavioral Sciences, 237, 1152-1159. https://doi.org/10.1016/j.sbspro.2017.02.171

Takahashi, R., \& Todo, Y. (2017). Coffee Certification and Forest Quality: Evidence from a Wild Coffee Forest in Ethiopia. World Development, 92, 158-166. https:// doi.org/10.1016/j.worlddev.2016.12.001

Teopista Akoyi , K., \& Maertens, M. (2017). Walk the Talk: Private Sustainability Standards in the Ugandan Coffee Sector. The Journal of Development Studies, 1792-1818 . https:// doi.org/10.1080/00220388.2017.1327663

Teopista Akoyia, K., Mitikub, F., \& Maertensa, M. (2020). Private sustainability standards and child schooling in the African coffee sector. Journal of Cleaner Production, 264, 1-24. https:// doi.org/10.1016/j.jclepro.2020.121713

Vanderhaegen, K., Teopista Akoyi, K., Dekonick, W. J., Muys, B., Verbist, B., \& Maertens, M. (2018). Do private coffee standards 'walk the talk' in improving socio-economic and environmental sustainability? Global Environmental Change, 51, 1-9. https://doi.org/10.1016/j.gloenvcha.2018.04.014

Vera Solano, J. A., \& Cañón Barriga, J. E. (2018). El valor agregado de un sistema de gestión ambiental más allá de la certificación. Bistua: Revista De La Facultad De Ciencias Basicas, 16(1), 86-91.

Weihrich, H., \& Koontz, H. (1993). Administración, una perspectiva global. México: McGraw-Hill. 